\title{
MSC-SEEDED DENSE COLLAGEN SCAFFOLDS WITH A BOLUS DOSE OF VEGF PROMOTE HEALING OF LARGE BONE DEFECTS
}

\author{
C. Gao ${ }^{1,2}$, E.J. Harvey ${ }^{1,3}$, M. Chua ${ }^{1,5}$, B.P. Chen ${ }^{1,4}$, F. Jiang ${ }^{1,3}$, Y. Liu ${ }^{6}$, A. Li ${ }^{1}$, H. Wang ${ }^{1}$ and J.E. Henderson ${ }^{1,2,3, *}$
}

${ }^{1}$ Bone Engineering Labs, ${ }^{2}$ Experimental Medicine, ${ }^{3}$ Experimental Surgery, Faculty of Medicine and ${ }^{4}$ Kinesiology \& Physical Education, Faculty of Education, McGill University, Montreal, Quebec, Canada

${ }^{5}$ Biotechnology Program, University of British Columbia, Burnaby, British Columbia, Canada

${ }^{6}$ Mathematics and Statistics, Queen's University, Kingston, Ontario, Canada

\begin{abstract}
The functional repair of large skeletal defects remains a significant challenge to orthopaedic surgeons due to the lack of effective strategies to promote bone regeneration, particularly in the elderly. This study investigated the potential use of bone marrow derived mesenchymal stromal cells (MSC) in a dense collagen scaffold with a bolus dose of vascular endothelial growth factor (VEGF) to repair a defect in the femoral diaphysis of mice. MSC isolated from bone marrow of 4-month-old donor mice were seeded in type I collagen gels that were then compressed to form scaffolds with a fibrillar density similar to osteoid. The cells remained metabolically active in scaffolds incubated in vitro for up to 15 days and differentiated into osteoblasts that deposited calcium-phosphate mineral into the scaffold, which was quantified using micro-computed tomographic (micro-CT) imaging. When implanted in a $1 \mathrm{~mm}$ x $3 \mathrm{~mm}$ unicortical defect the MSC-loaded scaffolds were rapidly mineralised and integrated into host bone with administration of $10 \mathrm{ng}$ of recombinant VEGF injected into the femoral canal at 4 days postoperative. Empty scaffolds and MSC-seeded scaffolds implanted in defects that did not receive a bolus dose of VEGF did not mineralise or integrate with native bone. The approach with MSC, hydrogels and a biologic factor already approved for human use warrants further pre-clinical investigation with a large animal model.
\end{abstract}

Keywords: Bone repair; collagen scaffold; mesenchymal stromal cells; vascular endothelial growth factor.

*Address for correspondence:

J.E. Henderson

Bone Engineering Labs

Research Institute-McGill University Health Centre

Surgical Research, C9.133, Montreal General Hospital

1650 Cedar Ave, Montreal, Quebec

Canada H3G 1A4

Telephone Number: 514-934-1934-44522

E-mail: janet.henderson@mcgill.ca

\section{Introduction}

Traumatic injury and excision of infected or neoplastic tissue all result in large defects that will not heal spontaneously, particularly in older individuals, and require an effective strategy for assisted repair to restore skeletal integrity and function (Nauth et al., 2011). The surgical reconstruction of large defects in bone requires an osteoinductive material to promote bone regeneration in the gap (Kolambkar et al., 2011). Autogenous bone grafts have been considered the gold standard for assisted repair under these circumstances due to their superior osteoinductive and osteoconductive properties. They are, however, limited in supply and lead to donor site morbidities that include pain and infection (Jones et al., 2006). Alternatively, de-vitalised allograft bone from cadaveric sources is more plentiful but has poor osteogenic capacity and carries the risk of acquired pathogens such as HIV or HBV (Joyce, 2005). Bone tissue engineering strategies using synthetic scaffolds, cells and soluble molecules may offer a better alternative to the use of human bone grafts to decrease morbidity and increase the potential for repair of large skeletal defects (Khan et al., 2008).

Bone is a nano-composite material with a $3 \mathrm{D}$ hierarchical structure that is composed of calcium phosphate mineral in a collagen matrix. Strategies that have been developed to overcome deficiencies in endogenous repair mechanisms involve the use of "smart scaffolds" as a delivery vehicle for cells and the growth factors they require for osteogenic differentiation. In this context it should be noted that the use of collagen sponges soaked with bone morphogenetic proteins (BMP, e.g., Medtronic INFUSE) to augment healing of large defects is being re-considered, due in part to questionable efficacy in clinical trials (Oetgen and Richards, 2010; Starman et al., 2012). Furthermore, serious side effects such as osteosarcoma, marrow fibrosis and ectopic bone formation have been associated with the use of recombinant bone morphogenetic protein (BMP). A variety of synthetic materials including degradable polymers, bioceramics (hydroxyapatite) and bioactive glass have also been developed for bone tissue engineering. Although these materials are approved for clinical use and are easy to fabricate with reproducible structure and mechanical properties, their early promise as temporary scaffolds for bone repair has not materialised. This is due primarily to their poor degradation property and replacement over time with mechanically sound and biologically functional bone. Alternative strategies under investigation 
Ex vivo

\begin{tabular}{|c|c|}
\hline \multicolumn{2}{|c|}{$\begin{array}{l}\text { Control \& MSC-seeded } \\
\text { dense collagen scaffolds } \\
\text { cultured up to } 15 \text { days in } \\
\text { differentiation medium }\end{array}$} \\
\hline & \\
\hline $\begin{array}{l}\mathrm{D} 5,10,15 \\
\text { netabolism, } \\
\text { ineralisation, } \\
\text { histology }\end{array}$ & $\begin{array}{c}\text { D } 6,9,12,15 \\
\text { RNA harvest } \\
\text { for molecular } \\
\text { markers }\end{array}$ \\
\hline
\end{tabular}

Ex vivo

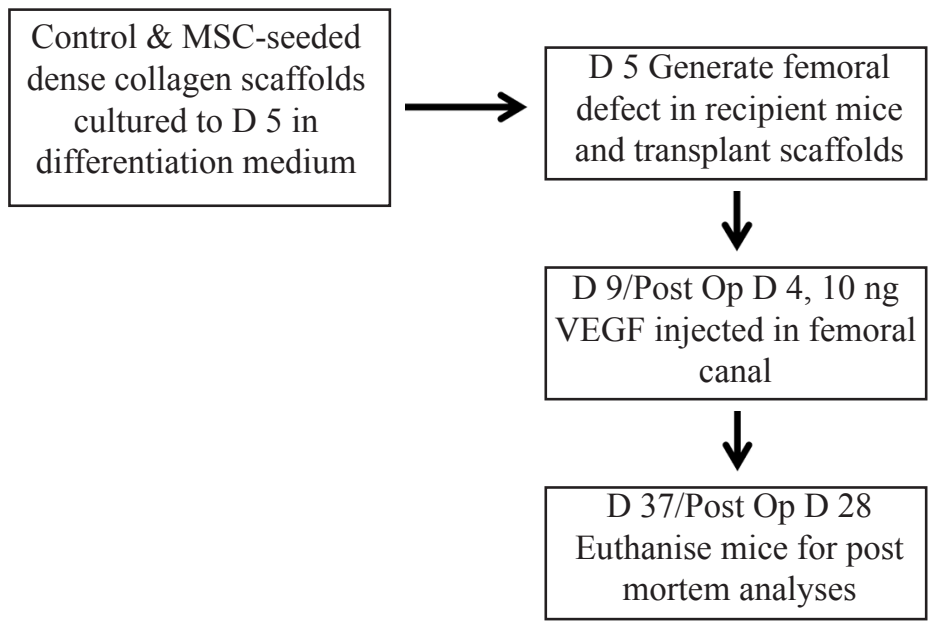

Fig. 1. Experimental design and work-flow for ex vivo and in vivo experiments. Mesenchymal stromal cells (MSC) were harvested from skeletally mature donor mice and seeded in hydrated type I collagen gels. Unconfined compression of the gels yielded dense collagen scaffolds that were cultured and removed at timed intervals for ex vivo evaluation of metabolic activity, mineralisation, histological analysis or for RNA isolation and molecular studies. For in vivo studies, uni-cortical defects were drilled in the femoral diaphysis of recipient mice and filled with scaffolds precultured for 5 days. Recipient mice were left for an additional 28 days for bone healing to take place.

involve replication of the hierarchical, porous structure of cancellous bone, with its nano- and meso-scale features, in synthetic scaffolds that enable the attachment and differentiation of bone forming cells and their precursors. Examples of patented technologies are 3D printed bioceramics (Gbureck et al., 2007) and porous titanium (LeNihouannen et al., 2008).

An alternative approach to ceramic scaffold-guided bone repair is the use of native collagen gels, reconstituted ex vivo to encase viable cells for subsequent transplantation into a bone defect. Type I collagen is a natural polymer and the primary component of the organic network into which hydroxyapatite crystals, the mineral phase of bone, are deposited (McKee and Sodek, 2000). It has been widely investigated for its potential use as a scaffold for bone tissue engineering due to its high osteoconductive capacity and low immunogenicity. Osteoblast-like cells (Brown et al., 2005) or mesenchymal stromal cells (MSC) isolated from whole bone marrow (Valverde-Franco et al., 2004) and seeded at high density in compressed collagen gels (Bitar et al., 2008) differentiated over time down the osteogenic lineage (Serpooshan et al., 2010). The "plastic compression" approach thus yields a Type I collagen matrix with a fibrillar density similar to that of bone matrix and containing viable osteogenic cells. Although these cell-seeded dense collagen scaffolds have been shown to support mineralisation by osteoblastic cell lines and MSC ex vivo, their capacity to repair bone in an appropriate pre-clinical model has not yet been demonstrated.

As is the case with bone development, bone regeneration is dependent on adequate and orderly recruitment of endothelial cells to form capillaries that will deliver oxygen, nutrients, growth factors and precursor cells to the site of healing (Kanczler and Oreffo, 2008). Vascular endothelial growth factor (VEGF) is a potent mediator of neo-vascularisation of the growth plates in developing bones (Amizuka et al., 2004), as well as angiogenesis that occurs during fracture repair (Street et al., 2002). The aim of the current study was to examine healing of a $1 \mathrm{~mm} x$ $3 \mathrm{~mm}$ surgically induced window defect in the mid femoral diaphysis of skeletally mature $\mathrm{C} 3 \mathrm{H}$ mice in response to MSC seeded dense collagen scaffolds. Integration of the scaffolds with native bone for optimal healing was promoted using a single, bolus dose of VEGF at a critical stage of callus formation.

\section{Materials and Methods}

Fig. 1 outlines the experimental approach and workflow chart for the study.

\section{Isolation of MSC and preparation of cell-seeded dense collagen scaffolds}

MSC were isolated essentially as described previously (Herbertson and Aubin, 1995). The soft tissue and the knee ends of the femora and tibiae were removed under aseptic conditions before placing the bones in Eppendorf tubes and centrifuging at low speed to dislodge the marrow. Bone marrow from individual 4-month-old wild type $\mathrm{C} 3 \mathrm{H}$ mice (total 3) was pooled, re-suspended in alpha Minimal Essential Medium (MEM) containing antibiotic/ antimycotic (Sigma-Aldrich, St. Louis, MO, USA) and $10 \%$ foetal bovine serum (FBS) (Wisent, St. Bruno, QC, Canada) and plated in $100 \mathrm{~mm}$ tissue culture dishes (Gao et al., 2012). At $80 \%$ confluence each dish yielded about $6 \times 10^{6}$ adherent cells, which were trypsinised and used at first passage to prepare 21 cell seeded scaffolds $\left(3 \times 10^{5}\right.$ / scaffold) using a modification of a previously described method (Serpooshan et al., 2010). A solution of $2.2 \mathrm{mg} /$ $\mathrm{mL}$ rat tail Type I collagen (First Link, West Midlands, UK) was diluted to $1.95 \mathrm{mg} / \mathrm{mL}$ with $10 \mathrm{x}$ Dulbecco's 
Modified Eagle's Medium (DMEM) (Sigma-Aldrich), and the $\mathrm{pH}$ adjusted to 7.5 with $\mathrm{NaOH}$. $200 \mu \mathrm{L}$ of alpha MEM (Sigma-Aldrich) containing $3 \times 10^{5} \mathrm{MSC}$ was added to each $800 \mu \mathrm{L}$ of $\mathrm{pH}$ adjusted collagen solution. The solution was then transferred into one well of a 24well plate (diameter $16 \mathrm{~mm}$ ) and incubated for $30 \mathrm{~min}$ at $37^{\circ} \mathrm{C}$ to complete gelation of the $16 \mathrm{~mm} \times 5 \mathrm{~mm}$ (depth) hydrated gels. Control scaffolds were prepared with $200 \mu \mathrm{L}$ of alpha MEM alone. Collagen gels were subjected to unconfined compression under a load of $1.4 \mathrm{kPa}$ for 3 min to expel water and generate the cellular or acellular (control) scaffolds measuring $16 \mathrm{~mm} \times 0.5 \mathrm{~mm}$ (depth) with an estimated collagen fibrillar density of $4.8 \mathrm{wt} \%$. Scaffolds were cultured in osteogenic alpha MEM supplemented with $10 \% \mathrm{FBS}, 50 \mu \mathrm{g} / \mathrm{mL}$ ascorbic acid, $10 \mathrm{mM} \beta$-glycerophosphate, with medium changes every 3 days for the duration of the experiment.

\section{Ex vivo metabolic activity and differentiation of MSC} in dense collagen scaffolds

Metabolic activity was quantified on 3 gels (technical replicates) seeded with MSC from each of the 3 different mice (biological replicates) at days 5, 10 and 15 (total 9 gels/mouse) of culture using the AlamarBlue ${ }^{\circledR}$ fluorescence assay according to the manufacturer's instructions (Life Technologies, Burlington, ON, Canada). After the metabolic assay the same scaffolds were fixed in $4 \%$ paraformaldehyde for $30 \mathrm{~min}$, washed in several changes of sterile phosphate buffered saline (PBS) and stored in PBS at $4{ }^{\circ} \mathrm{C}$ for micro-CT imaging. Scaffolds were imaged with a Skyscan 1172 micro-CT instrument (Skyscan, Kontich, Belgium) with no filter, a spatial resolution of $10 \mu \mathrm{m}$, a voltage of $50 \mathrm{kV}$ and power of $10 \mathrm{~W}$. Skyscan software was used for cross sectional reconstructions with NRecon, quantitative analysis with CTAn and $3 \mathrm{D}$ reconstruction with CTVol. Mineral content of the scaffolds was defined by setting the segmentation threshold between 120 and 255 in the binary mode of CTAn. The total volume of the scaffold and the volume of mineral in a defined region of interest (ROI) were quantified in 3 biological replicates at the indicated times. After micro-CT analysis, the cell seeded scaffolds were embedded in paraffin and consecutive $5 \mu \mathrm{m}$ sections stained for alkaline phosphatase (ALP) to assess osteoblast differentiation, Von Kossa/ Toluidine Blue to identify phosphate and Alizarin Red to identify calcium deposited in the collagen matrix.

For molecular analyses, RNA was extracted with Trizol ${ }^{\circledR}$ reagent (Invitrogen/Life Technologies Burlington, ON, Canada) used according to the manufacturer's instructions, on 3 technical replicates from each of the 3 different mice on days 6, 9, 12 and 15 (total 12 gels/ mouse) of culture. Each scaffold was homogenised in $1000 \mu \mathrm{L}$ of Trizol using an RNase-free pestle and mortar on ice. $250 \mu \mathrm{L}$ of chloroform was then added and the mixture centrifuged at $11,000 \mathrm{rpm}$ for $15 \mathrm{~min}$. About $500 \mu \mathrm{L}$ of supernatant was transferred into clean RNase-free tubes and mixed with an equal volume of $70 \%$ ethanol to precipitate the RNA. The RNA was then loaded onto an RNeasy Mini Kit (Qiagen, Toronto, ON, Canada) column and purified according to the manufacturer's instructions. The RNA extracted from cells in a single gel was diluted in $35 \mu \mathrm{L}$ of RNase-free water for qPCR analysis. Reverse transcription was performed using a high capacity kit and expression of marker genes quantified using the Taqman assay (Applied Biosystems/ Life Technologies, Burlington, ON, Canada). Expression of Type I collagen (Collagen I), Cbfa1, Osteocalcin, Osterix, PTH1R, and MMP13 were normalised to that of the housekeeping gene glyceraldehyde 3-phosphate dehydrogenase (GAPDH) and presented as fold increase over day 6 values.

\section{In vivo model to evaluate bone repair in response to cell seeded scaffolds}

Dense collagen scaffolds were prepared with or without MSC and cultured for 5 days in osteogenic medium prior to being trimmed, folded and implanted into freshly drilled femoral defects. All animal procedures were performed in strict accordance with a protocol approved by the McGill Facility Animal Care Committee, in keeping with the guidelines of the Canada Council on Animal Care. Rectangular window defects measuring $1 \mathrm{~mm} \times 3 \mathrm{~mm}$ were drilled using a $1 \mathrm{~mm}$ bit (Maxtech Consumer Products, Waterloo, ON, Canada) in the lateral surface of the femoral diaphysis of 8-10 month old male or female $\mathrm{C} 3 \mathrm{H}$ wild type mice. Where indicated, $10 \mu \mathrm{L}$ of sterile PBS containing $5 \times 10^{-8} \mathrm{M}$ recombinant mouse VEGF (R\&D Systems, Minneapolis, MN, USA) was introduced into the femoral canal at the level of the defect using a Hamilton syringe. Animals were randomised into one of the following groups: (1) no treatment $(n=8)$; (2) dense collagen scaffold implant on day $0(n=4)$; (3) cell seeded scaffold on day $0(n=8)$; (4) VEGF injection on post-operative day $4(n=10)$; (5) cell seeded scaffold on day $0+10$ ng VEGF on day 4 (n10). The mice were maintained post op with free access to food and water for 28 days, when they were euthanised and the femora harvested, fixed in $4 \%$ paraformaldehyde overnight, washed in several changes of PBS and stored in PBS at $4{ }^{\circ} \mathrm{C}$ for micro-CT and histological analyses.

\section{Quantitative micro-CT analysis of bone repair in response to cell seeded scaffolds}

Post mortem analyses were performed according to published protocols (Henderson et al., 2012). Intact femurs were first imaged using digital X-ray (Kubtec, Milford, CT US) before trimming and scanning with the Skyscan 1172 micro-CT using an Al0.5 filter with a spatial resolution of $5.5 \mu \mathrm{m}$ at a voltage of $50 \mathrm{kV}$ and power of $10 \mathrm{~W}$. The image dataset was analysed using NRecon, CTAn, and CTVol programs as described above for the mineralised scaffolds. In CTAn, a rectangular ROI measuring $2.9 \mathrm{~mm}$ x $0.9 \mathrm{~mm}$ x $0.77 \mathrm{~mm}$ was defined to cover the area of the window defect where the scaffold was placed and where repair took place. Quantification of bone mass and structural properties within the ROI was reported by CTAn in numeric format. These included percentage bone volume (BV/TV), bone mineral density (BMD), trabecular number (Tb.N), trabecular separation (Tb.Sp), trabecular thickness (Tb.Th), trabecular pattern factor (Tb.Pf), structural model index (SMI) and degree of anisotropy (DA). 

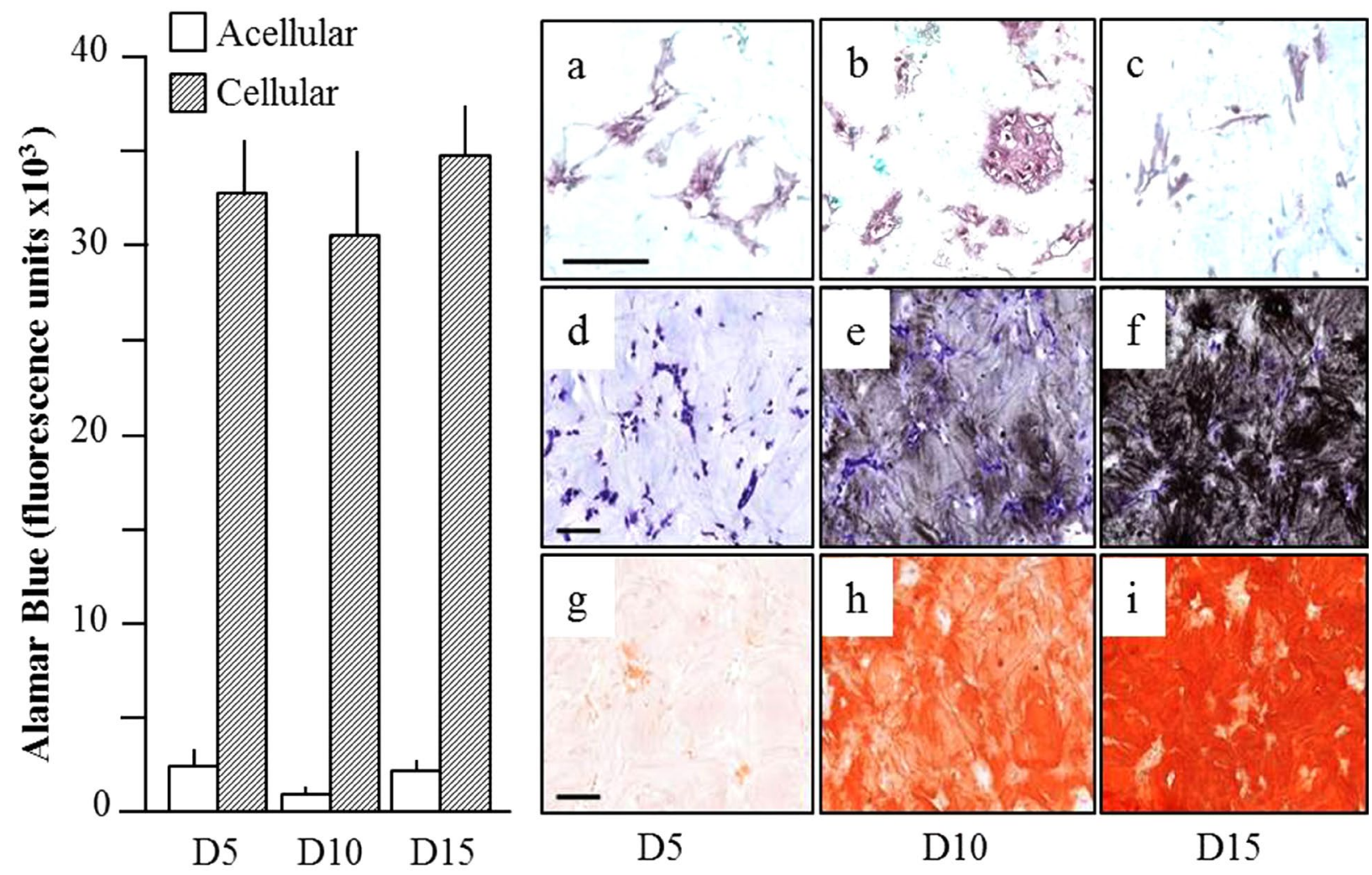

Fig. 2. Ex vivo growth and differentiation of MSC in dense collagen scaffold. MSC were isolated from whole bone marrow of wild type mice by adherence to plastic and diluted at a concentration of $300,000 / \mathrm{mL}$ in $1.58 \mathrm{mg} / \mathrm{mL}$ Type I collagen solution. Hydrated collagen gels were subjected to a compression force of $1.4 \mathrm{KPa}$ for 3 min and cultured in osteogenic medium. Alamar Blue was used to test the metabolic activity of cultures and 3 replicate gels removed at 5 days $(\mathbf{a}, \mathbf{d}, \mathbf{g}), 10$ days $(\mathbf{b}, \mathbf{e}, \mathbf{h})$ or 15 days $(\mathbf{c}, \mathbf{f}, \mathbf{i})$ for histological analysis. Sections of paraffin embedded gels were stained with alkaline phosphatase (a-c) to monitor MSC differentiation, with von Kossa/Toluidine Blue (d-f) to identify phosphate or Alizarin red (g-i) to identify calcium deposited in the collagen scaffold. Scale bars represent $100 \mu \mathrm{m}$.

\section{Histological analysis of bone repair in response to cell seeded scaffolds}

After micro-CT imaging the femurs were processed for histological analyses essentially as described previously (Valverde-Franco et al., 2004). Un-decalcified bones were embedded at low temperature in PMMA and serial $5 \mu \mathrm{m}$ sections were stained with $5 \%$ silver nitrate (Von Kossa) and counterstained with $0.2 \%$ toluidine blue to distinguish mineral from soft tissue. Adjacent sections were stained with Naphthol AS-TR phosphate (Sigma-Aldrich) in Trismaleate buffer $\mathrm{pH} 9.3$ to identify alkaline phosphatase (ALP) activity in osteoblasts or with disodium Naphthol AS-TR phosphate, sodium nitrite and pararosaniline hydrochloride in acetate buffer $\mathrm{pH} 5.0$ to identify tartrate resistant acid phosphatase (TRAP) activity in osteoclasts. A second group of bones was decalcified in $10 \%$ EDTA and embedded in paraffin for immunohistochemical staining of CD34 (Goat anti rat antibody, 1:300 dilution, R\&D Systems) positive vascular endothelial cells or osteocalcin (Goat anti mouse antibody, 1:400 dilution, Biomedical Technologies, Stoughton, MA, USA) positive osteoblasts. Sections were counter-stained with methyl green.

\section{Statistical analysis}

Ex vivo assays (Figs. 2 and 3, Table 1) were performed on 3 cell-seeded scaffolds from 3 biological replicates at each time point. In vivo data (Fig 4) is representative of 4 animals in group 2 (empty scaffold) and 8 to 10 biological replicates in all other groups. SPSS (IBM, Armonk, NJ, USA) was used for global analysis of variance (ANOVA) with posthoc Tukey's "honest significant difference" (HSD) test to determine significance with a probability of $p<0.05$.

\section{Results}

\section{Ex vivo characterisation of MSC-seeded dense collagen scaffolds}

In previous work, the hydraulic permeability of cell seeded dense collagen scaffolds was investigated and showed that increasing collagen density correlated with increased potential for the differentiation of MSC trapped in the fibrillar network (Serpooshan et al., 2010). In the present study, several modifications were made in the production of the dense collagen scaffolds (see Materials and Methods) 

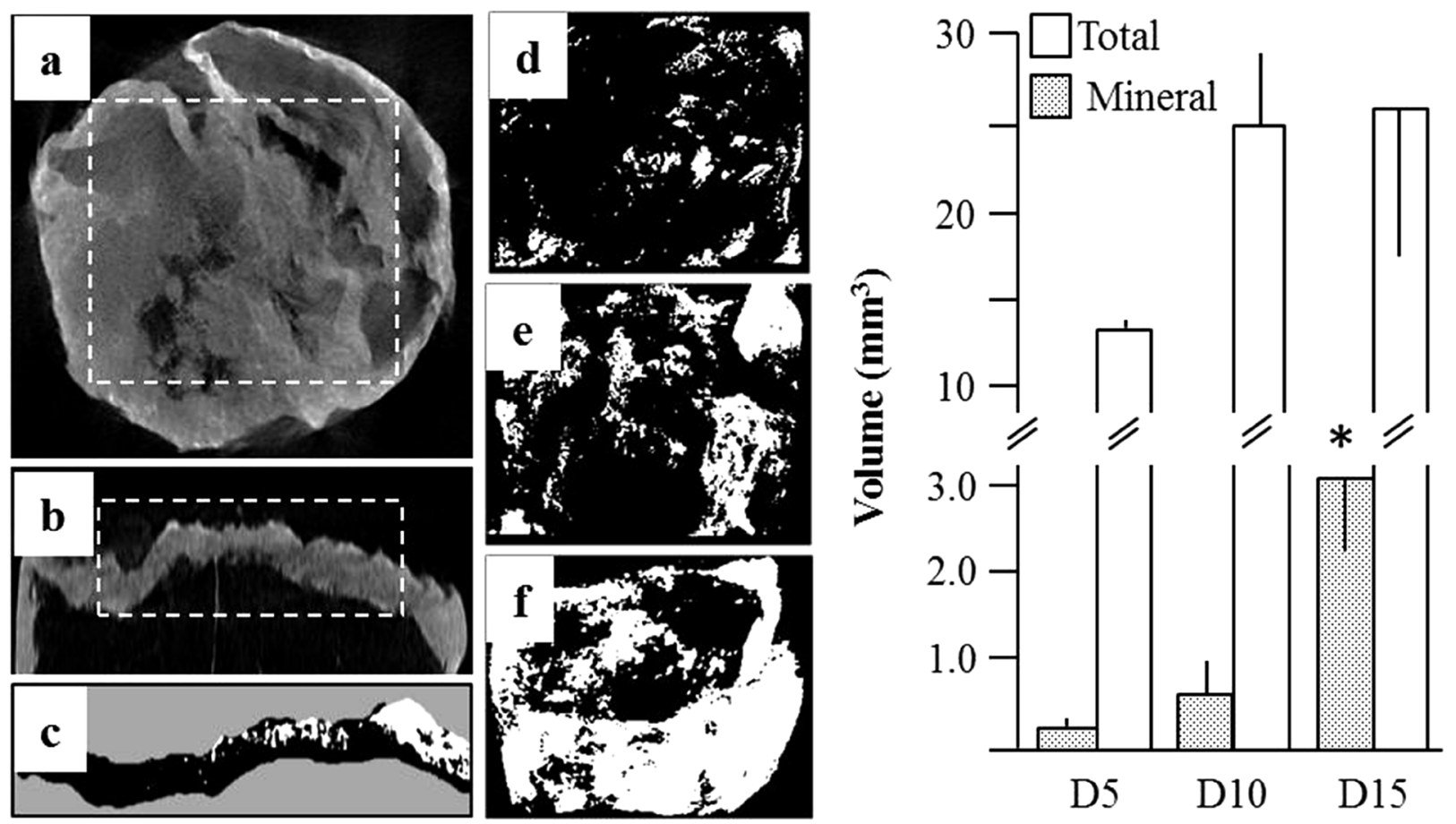

Fig. 3. Quantitative micro-CT analysis of scaffold mineralisation. Dense collagen scaffolds seeded with MSC were prepared and cultured as in Fig. 2, and 3 replicate scaffolds were harvested on day 5, 10 and 15 for micro-CT analysis of mineral deposition. The $5 \mathrm{~mm}$ x $5 \mathrm{~mm}$ volume of interest (VOI) is shown on a 3D reconstruction of a scaffold in the horizontal (a) and vertical $(\mathbf{b}, \mathbf{c})$ planes. Mineral content is shown in white on representative 3D images, binarised in CTAn, in the vertical plane (c) and in the horizontal plane on day 5 (d), day 10 (e) and day 15 (f). Quantitative data, expressed as the mean $\pm \mathrm{SD}$ of three replicate scaffolds, for total scaffold volume compared with the mineralised volume is shown for the different time points. Significantly different from day $5 * p<0.05$.

Table 1: qPCR analysis of gene expression in MSC-seeded dense collagen scaffolds cultured in osteogenic medium.

\begin{tabular}{|l|c|c|c|c|}
\hline Gene & Day 6 & Day 9 & Day 12 & Day 15 \\
\hline Cbfa1 & $1.01 \pm 0.16$ & $0.73 \pm 0.06^{\mathrm{b}}$ & $0.62 \pm 0.13^{\mathrm{b}}$ & $1.20 \pm 0.10^{\mathrm{a}}$ \\
\hline Collagen I & $1.01 \pm 0.14$ & $1.21 \pm 0.23$ & $2.86 \pm 0.13^{\mathrm{b}}$ & $2.06 \pm 0.57^{\mathrm{b}}$ \\
\hline MMP13 & $1.01 \pm 0.12$ & $0.57 \pm 0.11^{\mathrm{b}}$ & $0.23 \pm 0.02^{\mathrm{b}}$ & $0.33 \pm 0.02^{\mathrm{b}}$ \\
\hline PTH1R & $1.00 \pm 0.06$ & $0.96 \pm 0.11$ & $2.27 \pm 0.16^{\mathrm{b}}$ & $2.02 \pm 0.43^{\mathrm{b}}$ \\
\hline Osteocalcin & $1.02 \pm 0.23$ & $2.40 \pm 0.77$ & $9.49 \pm 4.59^{\mathrm{b}}$ & $13.30 \pm 3.20^{\mathrm{b}}$ \\
\hline Osterix & $1.01 \pm 0.17$ & $1.19 \pm 0.19$ & $2.14 \pm 0.50^{\mathrm{b}}$ & $2.18 \pm 0.41^{\mathrm{b}}$ \\
\hline
\end{tabular}

One-way ANOVA was used to analyse the differences in gene expression of cells embedded in dense collagen scaffolds harvested at $6,9,12$ or 15 days of culture in osteogenic medium. Expression at day 9, 12 and 15 was compared with that at day 6 using Tukey's HSD test. Significantly different from day $6{ }^{\mathrm{a}} p<0.05^{\mathrm{b}} p<0.01$.

to optimise the differentiation of the MSC and generate a scaffold with greater mechanical stability for in vivo manipulation. The reduction of AlamarBlue ${ }^{\circledR}$ reagent to a fluorescent red product by metabolically active cells embedded in the dense collagen scaffolds indicated no change in their viability for up to 15 days when cultured in osteogenic medium (Fig. 2, graph). Sections of paraffin embedded gels stained for ALP (Fig. 2a-c) showed little change in activity between day 5 (Fig. 2a) and day 10 (Fig. 2b) but then a significant decrease was seen by day 15 (Fig. 2c). In contrast, adjacent sections stained with Von Kossa/toluidine blue showed a progressive increase in phosphate deposition (Fig. 2d-f), which was accompanied by increased Alizarin Red staining of calcium (Fig. 2g-1), in the absence of any apparent change in scaffold cellularity, as evidenced by haematoxylin and eosin stained histological sections (data not shown). Quantitative PCR analysis (Table 1) of RNA extracted from the embedded cells after $6,9,12$ or 15 days showed a time dependent up-regulation of markers of osteoblast differentiation, including type 1 collagen, the receptor for parathyroid hormone related protein (PTH1R), the matrix protein osteocalcin and the transcription factor osterix. Expression of the transcription factor Cbfa1 and matrix 

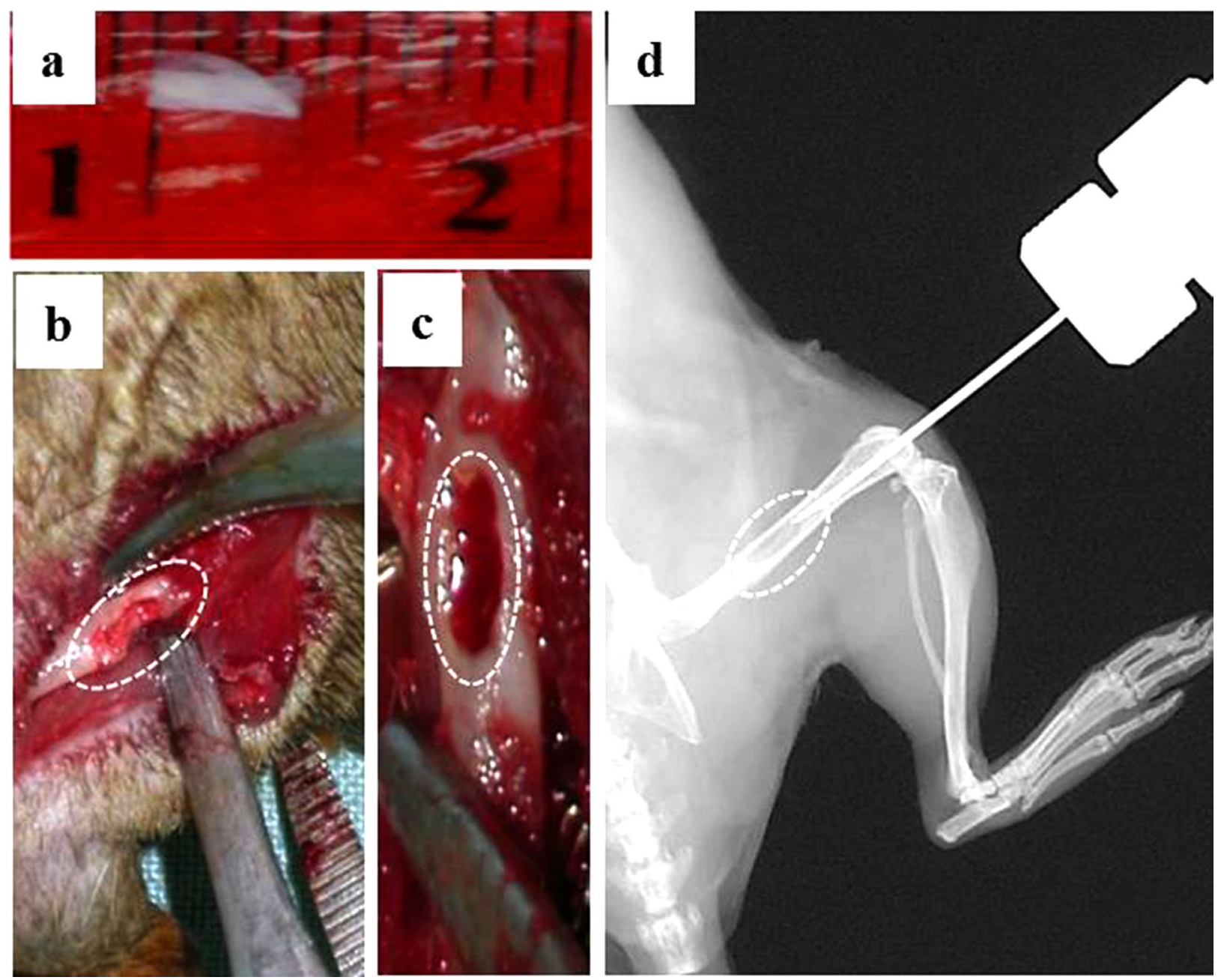

Fig. 4. Femoral window defect and VEGF administration. MSC-seeded scaffolds prepared as described in Fig. 2 and cultured ex vivo for 5 days were loosely folded (a) before placing carefully in $1 \mathrm{~mm} \times 3 \mathrm{~mm}$ window defects drilled into the mid-diaphysis of the femur in 8-10 month old wild type $\mathrm{C} 3 \mathrm{H}$ mice (b-c). On post-operative day 4 , $10 \mu \mathrm{L}$ of PBS containing $10 \mathrm{ng}$ of recombinant mouse VEGF was injected into the intramedullary canal (d) via the inter-condylar notch using a Hamilton syringe. Femora were harvested for analyses at post-operative day 28.

metalloproteinase 13 decreased over the same timeframe, but with a significant increase in Cbfa1 on day 15. Taken together with the increase in matrix phosphate and calcium, these results suggested a progressive increase in mineral deposition by osteogenic cells that had differentiated from the MSC entrapped in the collagen scaffold.

A program written in the custom processing mode of CTAn software was used to delineate a region of interest (ROI) on the 2D reconstructed images of the scaffolds, shown in horizontal (Fig. 3a) and vertical (Fig. 3b,c) planes, for quantification of mineral based on differential X-ray attenuation in scaffolds cultured for 5, 10 or 15 days (Fig. $3 \mathrm{~d}-\mathrm{f}$ ). The quantitative data, shown in the graph, reveals an increase in the scaffold volume/thickness between day 5 and day 10 and no change thereafter. In contrast, the mineral content increased steadily over time to reach a more than 14-fold increase on day 15 over day 5 .
Bone repair in response to implantation of MSCseeded dense collagen scaffold in vivo

Data from a previous study (Serpooshan et al., 2010) and from the in vitro data from this study indicated that day 5 of culture marked decreased proliferation and the onset of differentiation of the MSCs seeded in scaffolds. To optimise conditions for bone repair, the scaffolds were therefore cultured for 5 days before loosely rolling and implanting them in $1 \mathrm{~mm} \times 3 \mathrm{~mm}$ drill-hole defects in the femoral mid-diaphysis of skeletally mature mice (Fig. 4ac). Given the critical role played by neovascularisation in bone repair, some of the defects were treated with a single dose of VEGF in the presence or absence of cell-seeded scaffolds at post-operative day 4 (Fig. 4d) and bone repair evaluated at post-operative day 28 (Fig. 5). High resolution plain X-ray images showed little difference between femurs receiving the different treatments (Fig. 5a-e). High 

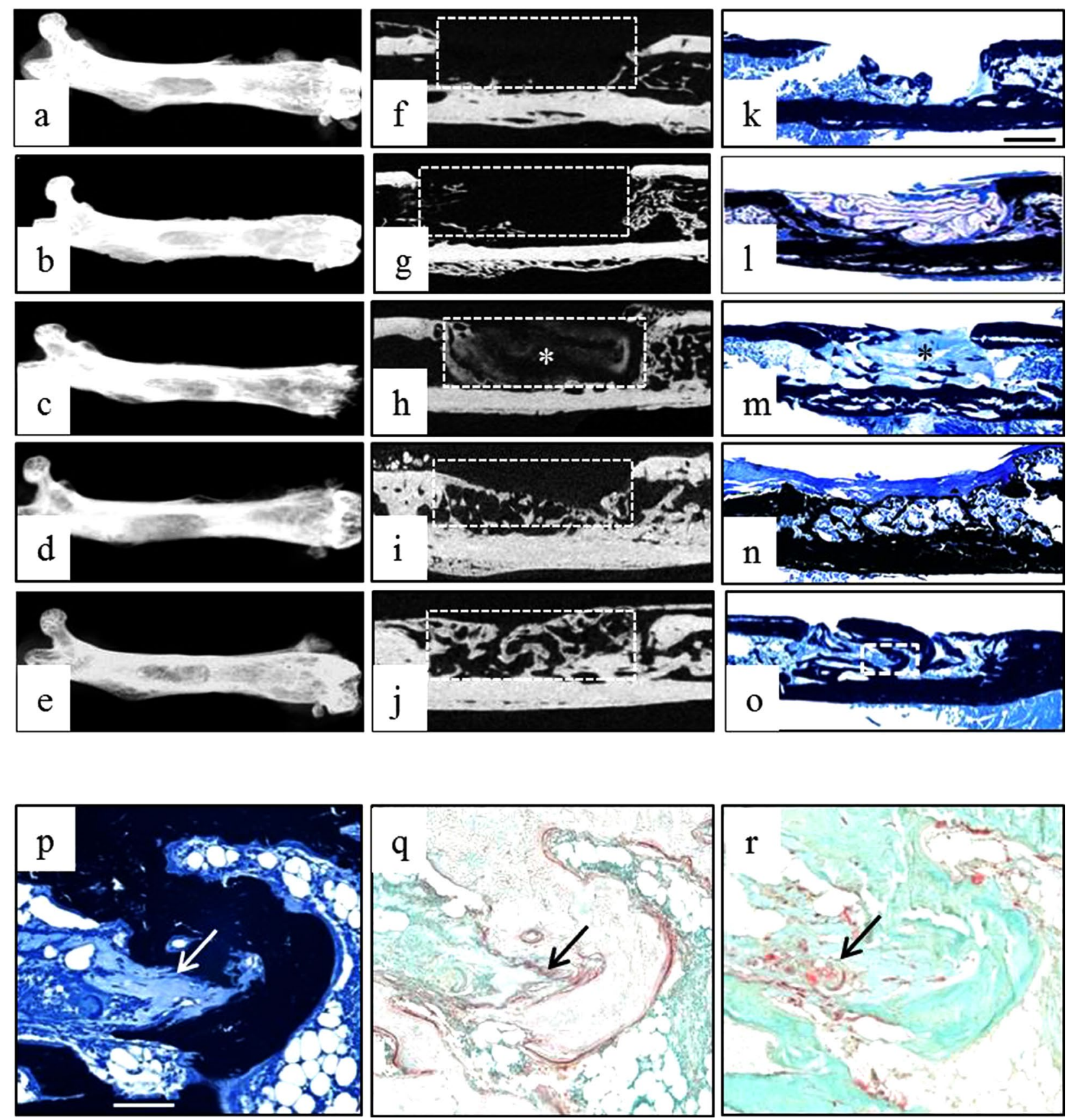

Fig. 5. Radiologic and histologic analyses of bone repair. Representative X-ray (a-e), micro-CT (f-j) and plastic embedded calcified bone stained with Von Kossa/Toluidine Blue (k-o) images are shown of bone repair at 28 days post-operative for defects receiving no treatment $(n=8 \mathbf{a}, \mathbf{f}, \mathbf{k})$, collagen scaffold alone $(n=4 \mathbf{b}, \mathbf{g}, \mathbf{l})$, MSC-seeded scaffold $(n=8 \mathbf{c}, \mathbf{h}, \mathbf{m}), 10$ ng VEGF alone $(n=10 \mathbf{d}, \mathbf{i}, \mathbf{n})$ or both MSC-seeded scaffold and VEGF $(n=10 \mathbf{e}, \mathbf{j}, \mathbf{o})$. The asterisk $(\mathbf{h}, \mathbf{m})$ indicates the rolled MSC-seeded scaffold and the white dotted line $(\mathbf{f}-\mathbf{j})$ delineates the $2.9 \mathrm{~mm} \mathrm{x}$ $0.9 \mathrm{~mm} \times 0.8 \mathrm{~mm}$ region of interest (ROI) for the quantitative micro-CT analyses shown in Fig 6 . The area contained within the white dotted line in (o) is shown at higher magnification (p), along with sections stained for alkaline phosphatase (ALP, q) and tartrate resistant acid phosphatase (TRAP, r). ALP positive osteoblasts and TRAP positive osteoclasts were identified at the junction (arrows) of un-mineralised and mineralised scaffold. Scale bars represent $500 \mu \mathrm{m}(\mathbf{k - o})$ and $50 \mu \mathrm{m}(\mathbf{p}-\mathbf{r})$.

resolution 2D micro-CT images (Fig. 5f-j) showed little healing of defects with no treatment (Fig 5f), those treated with an empty dense collagen scaffold (Fig. $5 \mathrm{~g}$ ) or with an MSC-seeded scaffold (Fig. 5h). There was some healing in the presence of VEGF (Fig. 5i) with significantly more in those defects treated with the combination of cell-seeded scaffold and VEGF (Fig. 5j). The corresponding thin sections of plastic embedded tissue stained with Von Kossa/ toluidine blue (Fig. 5k-o) confirm significant accumulation of mineral only in the defects treated with VEGF in the absence (Fig. 5n) or presence (Fig. 5o) of MSC. The boxed region in Fig 5o is shown at higher magnification in Fig. $5 \mathrm{p}$. Adjacent sections stained for alkaline phosphatase (ALP, Fig. 5q) or tartrate resistant acid phosphatase (TRAP, 

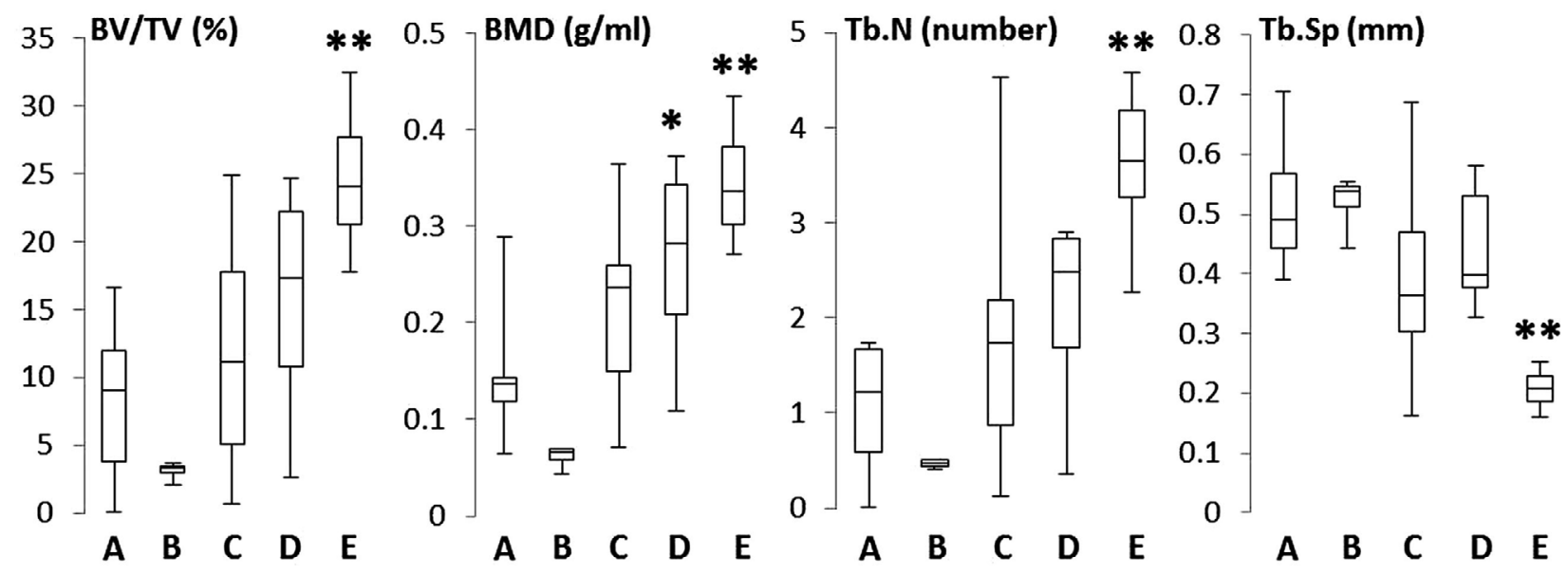

Fig. 6. Quantitative micro-CT analysis of bone repair. All femora were scanned on a Skyscan 1172 instrument with a spatial resolution of $5.5 \mu \mathrm{m}$. Using CTAn analytical software, bone parameters were quantified in a rectangular region of interest (ROI) measuring $2.9 \mathrm{~mm}$ x $0.9 \mathrm{~mm}$ x $0.8 \mathrm{~mm}$ occupied by the scaffold. Values for bone as a \% of tissue volume (BV/TV), bone mineral density (BMD), trabecular number (Tb.N) and trabecular separation (Tb.Sp) are shown for femora receiving no treatment (A), empty collagen scaffold (B), MSC-seeded scaffold (C), VEGF alone (D) or MSC-seeded scaffold and VEGF (E). Significantly different from A * $p<0.05 ; * * p<0.001$.

Fig. 5r) show significant ALP activity in osteoblasts and numerous TRAP positive osteoclasts at the junction of mineralised and un-mineralised scaffold (arrows).

Quantitative analysis of 3D micro-CT data (Fig. 6) revealed a significant improvement in bone volume relative to tissue volume (BV/TV), bone mineral density (BMD), trabecular number (Tb.N) and trabecular separation (Tb. $\mathrm{Sp}$ ) in the group receiving the combination of cell-seeded scaffold and a single post-operative dose of VEGF (Fig. 6e) compared with the control femurs receiving no treatment (Fig. 6a). The differences in these parameters did not reach significance for the other treatment groups except for an increase in BMD in response to a single dose of VEGF. There were no significant differences (data not shown) in trabecular thickness (Tb.Th) or in indices of bone architecture including degree of anisotropy (DA), trabecular pattern factor (Tb.Pf) or structure model index (SMI).

To further explore the increase in mineral content of the defects treated with cell-seeded scaffolds and a bolus dose of VEGF, representative specimens were decalcified and embedded in paraffin for immunohistochemical analyses (Fig. 7). Sections stained for CD34 (Fig. 7a-d), which is selective for haematopoietic progenitors and the endothelium of small vessels, revealed numerous positive cells within bone marrow adjacent to regenerating bone in animals that received no treatment (Fig. 7a boxed area and $\mathrm{a}_{1}$ ). CD34 positive cells were significantly increased in mice that received a bolus dose of VEGF alone (Fig. 7c and $c_{1}$ ). There was a noticeable absence of CD34 positive cells in the specimens containing an empty scaffold (Fig. 7b and $b_{1}$ ) but they were seen amongst the folds of the scaffold in animals treated with MSC-seeded scaffolds and VEGF (Fig. $7 \mathrm{~d}_{\text {and }} \mathrm{d}_{1}$ ). Osteocalcin is a non-collagenous protein that has been implicated in tissue mineralisation and is deposited in bone matrix by mature osteoblasts. Minimal staining for osteocalcin was evident at the scaffold/bone interface in specimens containing an empty scaffold (Fig. 7e and $\mathrm{e}_{1}$ ). However, osteocalcin staining was seen deposited between the folds of the cell-seeded scaffold (Fig. $7 \mathrm{f}_{1}$, asterisk) and at the junction of the mineralising scaffold and native bone (Fig. $7 \mathrm{f}_{1}$, arrows).

\section{Discussion}

Bone repair in the adult skeleton begins with a prototypical inflammatory response to injury during which MSC are recruited from the sub-periosteum, the bone marrow and adjacent soft tissue to form a soft callus (Gerstenfeld et al., 2003). During the consolidation stage, MSC in this granulation tissue differentiate under the influence of local cytokines and growth factors into cells that form new trabecular and cortical bone. The number of cells and bioactive factors required for bone regeneration decline with age, in chronic disease or following treatment for inflammatory or neoplastic disease, which compromises the repair process and leads to bone necrosis and non-union of fractures (Gruber et al., 2006). An extensive literature has accumulated over several decades that documents attempts to promote bone regeneration using scaffolds, cells, cytokines and growth factors. A recurrent theme throughout this literature is the need for in vivo validation of novel therapeutic approaches in clinically relevant models.

Genetically modified and inbred strains of mice have been used with success for more than three decades to map the regulatory pathways involved in bone development and skeletal metabolism and many of these pathways are recapitulated during bone regeneration in the adult. Despite 

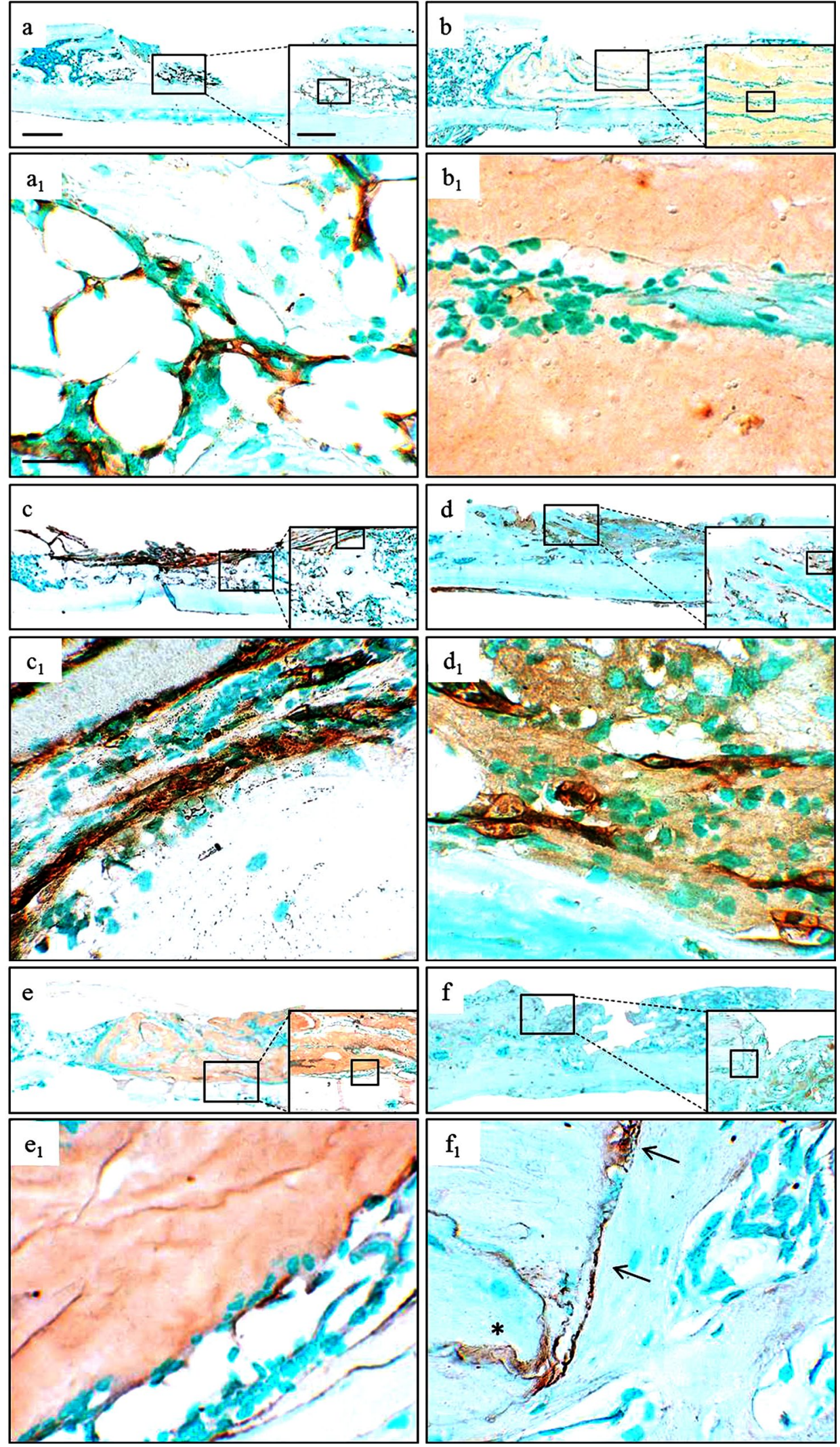

Fig. 7. Immunohistochemical analysis of vascularity and bone formation. Sections of decalcified, paraffin embedded bones were stained with CD34 antiserum (a-d) to identify vascular endothelial cells or osteocalcin antiserum (e-f) to identify osteoblasts. Vascular endothelial cells (brown stain) were localised in regenerating bone on representative specimens with no treatment ( $\mathbf{a}$ and $\mathbf{a}_{1}$ ), those receiving VEGF alone ( $\mathbf{c}$ and $\mathbf{c}_{1}$ ) and those treated with MSC-seeded scaffold and VEGF (d and $\mathbf{d}_{1}$ ) but not in specimens receiving collagen scaffold alone (b and $\mathbf{b}_{1}$ ). A few osteocalcinpositive cells were seen at the periphery of the dense collagen scaffold $\left(\mathbf{e}_{1}\right)$ with more extensive deposits within the scaffold (asterisk) and at the bone-scaffold interface (arrows) in specimens treated with MSC-seeded scaffold and VEGF $\left(\mathbf{f}_{1}\right)$. Boxed areas in the insets indicate regions shown at higher magnification in the corresponding panels marked with “" ". Scale bars represent $500 \mu \mathrm{m}$ (main panel-f); $200 \mu \mathrm{m}$ (insets) and $20 \mu \mathrm{m}$ (large images). 
their small size, this extensive body of knowledge has identified mice as a preferred species for in vivo analyses of bone repair (Karsenty and Wagner, 2002). A mouse model of a segmental defect with external fixation (Tay et al., 1998) has been used extensively to model nonunion but confounding factors arising from high mobility made it unsuitable for study. We therefore modified a cortical drill-hole defect (Monfoulet et al., 2010) to generate a reproducible, mechanically stable defect, where both cortical bone and endogenous bone marrow were disrupted. The higher bone mineral content, density and biomechanical strength of cortical bone in $\mathrm{C} 3 \mathrm{H}$ mice compared with other mouse strains (Akhter et al., 2000) allows for generation of a $1 \mathrm{~mm}$ x $3 \mathrm{~mm}$ uni-cortical defect that is stabilised by the remaining cortex and will not heal without therapeutic intervention (Gao et al., 2011). The study builds on previous work that characterised the ex vivo differentiation of MSC in a dense collagen scaffold (Serpooshan et al., 2010) by investigating the potential of cell-seeded scaffolds for bone healing in vivo. The results indicate that MSC seeded dense collagen scaffolds optimised for the osteogenic differentiation of MSC and then transplanted into femoral window defects will mineralise over time and integrate with native bone in the presence of VEGF.

An ideal scaffold for bone tissue engineering should be osteoinductive, osteoconductive and subject to resorption and replacement by functional site-specific bone over time (Harvey et al., 2010). Hydroxyapatite-tricalcium phosphate (HA-TCP), alone or in combination with autogenous bone, has been under investigation for more than three decades for this purpose (Rootare et al., 1978; Moore et al., 1987). More recent studies have focused primarily on the use of calcium and magnesium based scaffolds and cements for the controlled release of bioactive molecules, including antibiotics and anti-neoplastic agents, into the bone micro-environment (Barralet et al., 2009; Roohani-Esfahani et al., 2012; Soundrapandian et al., 2010). A major disadvantage of calcium phosphate based scaffolds is resistance to resorption by osteoclasts, unless they are pre-treated with an osteoclast activator such as RANK ligand (LeNihouannen et al., 2008), which has severely limited their clinical utility. Modification of their surface composition and topography with silk, which has a fibrillar structure similar to that of native collagen, has improved both the mechanical properties and biocompatibility of biphasic calcium phosphate scaffolds (Roohani-Esfahani et al., 2012). However, the increase in manufacturing complexity of composite scaffolds raises the cost considerably, which will ultimately be passed on to consumers in the healthcare industry.

To avoid the problems associated with traditional ceramic scaffolds and to develop a mechanism for introducing osteogenic cells into poor quality bone, a natural polymeric scaffold that resembles the organic or "osteoid" phase of native bone was selected for the current studies. Due to their inherent fragility, hydrated type I collagen scaffolds are not commonly associated with hard tissue engineering applications but are commercially available for soft tissue engineering, most notably skin. In 2004 Professor R.A. Brown at University College,
London developed a dense cellular scaffold with useful mechanical properties and ultrastructure simply by rapid expulsion of fluid from hydrated collagen gels using plastic compression (Brown et al., 2005). In an ex vivo study we recently demonstrated long-term viability and osteogenic differentiation of MSC in dense Type I collagen scaffolds (Serpooshan et al., 2010). The ultra-rapid engineering and prior clinical approval for the use of collagen-based wound dressings make this an attractive option for bone tissue engineering. For the current application of cortical bone healing, pilot studies were conducted with variable compressive force and time, as well as different MSC seeding densities to generate a construct with optimal osteogenic properties. To enhance the mechanical properties of the scaffold while maintaining adequate diffusion of oxygen and nutrients, the compressive force and time were first increased by about $30 \%$ while maintaining the MSC concentration at $6 \times 10^{6} / \mathrm{mL}$ dense collagen. This led to minimal scaffold mineralisation when implanted in the murine window defect and effectively formed a barrier to the influx of endogenous cells at postoperative 4 weeks. Based on previous ex vivo work with osteogenic MG-63 osteosarcoma cells (Bitar et al., 2008), a seeding density of $3 \times 10^{5} \mathrm{MSC} / \mathrm{mL}$ of hydrated collagen was selected for a final concentration of $3 \times 10^{7} / \mathrm{mL}$ dense collagen.

In our ex vivo studies, this construct with a 5 fold increase in cell numbers and improved mechanical properties, supported the long term metabolic activity and differentiation of MSC into osteoblasts that deposited a calcium-phosphate rich mineral in the extracellular matrix. Increased expression of recognised molecular markers of osteoblast differentiation (type I collagen, PTH1R, ostecalcin, osterix) were accompanied by the reported reduction of Cbfal and MMP13 up to Day 12, with an increase on day 15 as reported previously (Aubin and Triffitt, 2002; Komori, 2010). Unfortunately, when implanted in the femoral window defects it acted as a barrier, separating the transplanted and endogenous cells. Insufficient vascularisation for effective mass transport of oxygen, nutrients and waste products through the scaffold has been documented as an obstacle to integration of cellseeded polymeric scaffolds into native bone in vivo (Lin et al., 2010). The angiogenic properties of VEGF are well recognised in both bone development (Carlevaro et al., 2000) and bone repair (Street et al., 2002; Uchida et al., 2003). It is a potent inducer of endothelial cell proliferation and migration and has been used in combination with a variety of biomaterials, including Type I collagen, to promote angiogenesis (Shen et al., 2008). When recombinant human (rh) VEGF was covalently linked to a commercial collagen sponge to achieve sustained release kinetics, it enhanced vascularisation and the repair of myocardium in vivo (Miyagi et al., 2011). We hypothesised that VEGF-induced angiogenesis would improve integration of the dense collagen scaffold with native bone in the healing window defect, in a manner similar to vascular invasion of the growth plate during long bone development (Amizuka et al., 2004). A single, intra-medullary therapeutic dose of VEGF was therefore administered at postoperative day 4 , which is the stage of fracture repair at which a haematoma has formed and 
an inflammatory response initiated. In the early phase of fracture repair a coagulation cascade is triggered and inflammatory cells migrate to the site where they release cytokines such as IL-1 and IL-6, and platelets also release important signalling molecules including PDGF and TGF- $\beta$. Although the mechanism of VEGF action under the present circumstances is not fully characterised, Fig 7 shows vascular endothelial cells and bone regeneration were increased at 28 days post-operative in the defects that received VEGF on day 4 post-operative. In the case of the defect that received both the cell-seeded scaffold and VEGF, it is possible that the inflammatory events in adjacent bone somehow modified the dense collagen scaffold. Alteration of the scaffold might allow tethering of the injected VEGF, thus protecting it from degradation and enabling a more sustained release. Alternatively, the VEGF could have been tethered by structural molecules such as fibrin in the haematoma and released later to induce neo-vascularisation (Backer et al., 2006; Zisch et al., 2001). In fact, Fig 7 shows quite clearly that VEGF acted synergistically with the cell-seeded dense collagen scaffold to induce bone formation.

The presence of mineralised scaffold in the centre of the defect and some remaining un-mineralised scaffold at the border with adjacent bone indicates that osteoinduction was initiated by cells trapped in the scaffold in an "inside out" manner. Of additional interest was the robust ALP staining within and at the periphery of the healing defect at 28 days in vivo whereas there was a decline in ALP activity ex vivo at 15 days. Together with the presence of TRAPpositive osteoclasts in and around the scaffold, this suggests active matrix turnover and release of signalling molecules that would promote osteogenic cell migration, vascular invasion and enhance mass transport. The osteoconductive properties of the mineralised scaffold, aided by VEGF, would thus enable migration and attachment of endogenous bone forming progenitor cells by way of the newly formed vessels. The presence of both osteoblasts and osteoclasts in central and peripheral areas, as well as osteocalcin positive "osteocyte-like" cells embedded in mineralised matrix, further suggests the extracellular matrix within the bone defect was undergoing robust turnover. Additional studies aimed at determining the fate of transplanted MSCs and their relationship with endogenous cells during fracture repair will require the use of GFP or other labelled cells in the scaffold. A detailed time course, with bone healing examined as early as 4 days, and at weekly intervals thereafter up to 8-12 weeks, in the presence and absence of VEGF therapy will be necessary to reveal the molecular mechanisms underlying the increase in bone formation at 4 weeks seen in the current study.

\section{Conclusion}

The results of our study indicate that a type I collagen scaffold with the consistency of un-mineralised bone induced the osteoblastic differentiation of MSC when folded into a large defect in the mouse femur. Deposition of mineral within the scaffold effectively filled the defect with "tissue" that resembled trabecular bone. A single post-operative dose of recombinant VEGF promoted neovascularisation and integration of the mineralised scaffold with endogenous bone that formed around the implant. The approach therefore represents a rapid, safe and cost effective mechanism for bone tissue engineering where endogenous mechanisms are compromised.

\section{Acknowledgements}

The authors gratefully acknowledge the assistance of Alison Butler (UBC Biotechnology Program), members of the RVH Orthopaedic Research Laboratory for assistance with RNA isolation and quantitative PCR measurements and Vahid Serpooshan (Nazhat Lab) for guidance with the plastic compression technique. The work was supported by grants from the Canadian Institutes of Health Research (CIHR) and by the Fonds de recherche en santé du Québec. M. Chua, A. Butler and B.P. Chen received summer studentships from the FRQ-S sponsored RSBO and Fan Jiang from McGill Faculty of Medicine. C. Gao was supported by studentship awards from CIHR-sponsored MENTOR, NCE-MITACS Accelerate, FRQS-sponsored RSBO, McGill MIDAS and the Research Institute of the McGill University Health Centre (RI-MUHC). The RI-MUHC is an FRQ-S sponsored Centre de Recherche.

\section{References}

Akhter MP, Iwaniec UT, Covey MA, Cullen DM, Kimmel DB, Recker RR (2000) Genetic variations in bone density, histomorphometry, and strength in mice. Calcif Tissue Intl 67: 337-344.

Amizuka N, Davidson D, Liu H, Valverde-Franco G, Chai S, Maeda T, Ozawa H, Hammond V, Ornitz DM, Goltzman D, Henderson JE (2004) Signalling by fibroblast growth factor receptor 3 and parathyroid hormone-related peptide coordinate cartilage and bone development. Bone 34: 13-25.

Aubin JE, Triffitt J (2002) Mesenchymal stem cells and the osteoblast lineage. In: Principles of Bone Biology (Bilezikian JP, Raisz LG, Rodan GA, eds), Academic Press, New York, pp 59-81.

Backer MV, Patel V, Jehning BT, Claffey KP, Backer JM (2006) Surface immobilization of active vascular endothelial growth factor via a cysteine-containing tag. Biomaterials 27: 5452-5458.

Barralet J, Gbureck U, Habibovic P, Vorndran E, Gerard C, Doillon CJ (2009) Angiogenesis in calcium phosphate scaffolds by inorganic copper ion release. Tissue Eng Part A 15: 1601-1609.

Bitar M, Brown RA, Salih V, Kidane AG, Knowles JC, Nazhat SN (2008) Effect of cell density on osteoblastic differentiation and matrix degradation of biomimetic dense collagen scaffolds. Biomacromolecules 9: 129-135.

Brown RA, Wiseman M, Chuo CB, Cheema U, Nazhat SN (2005) Ultrarapid engineering of biomimetic materials and tissues: fabrication of nano- and microstructures by plastic compression. Adv Funct Mater 15: 1762-1770. 
Carlevaro MF, Cermelli S, Cancedda R, Cancedda FD (2000) Vascular endothelial growth factor (VEGF) in cartilage neovascularization and chondrocyte differentiation: autocrine-paracrine role during endochondral bone formation. J Cell Sci 113: 59-69.

Gao C, Nguyen O, Serpooshan V, El Chaarani B, Nazhat SN, Harvey EJ, Henderson JE (2011) Rodent model of adult stem cell transplantation for bone repair. J Bone Joint Surg Br 93-B suppl IV: 553.

Gao C, Seuntjens J, Kaufman GN, Tran-Khan N, Butler A, Li A, Wang H, Buschmann MD, Harvey EJ, Henderson JE (2012) Mesenchymal stem cell transplantation to promote bone healing. J Orthop Res 30: 1183-1189.

Gbureck U, Holzel T, Doillon CJ, Muller FA, Barralet JE (2007) Direct printing of bioceramic implants with spatially localised angiogenic factors. Adv Mater 19: 795 800.

Gerstenfeld LC, Cullinane DM, Barnes GL, Graves DT, Einhorn TA (2003) Fracture healing as a post natal developmental process: molecular, spatial, and temporal aspects of its regulation. J Cell Biochem 88: 873-884.

Gruber R, Koch H, Doll BA, Tegtmeier F, Einhorn TA, Hollinger JO (2006) Fracture healing in the elderly patient. Exp Gernotol 41: 1080-1093.

Harvey EJ, Henderson JE, Vengallatore ST (2010) Nanotechnology and bone healing. J Ortho Trauma 24: $\mathrm{S} 25-\mathrm{S} 30$.

Henderson JE, Gao C, Harvey EJ (2012) Skeletal phenotyping in rodents. In: Osteoporosis Research: Animal Models (Duque G, Watanabe K, eds), Springer-Verlag, London, pp. 13-28.

Herbertson A, Aubin JE (1995) Dexamethasone alters the sub-population makeup of bone marrow stromal cultures. J Bone Miner Res 10: 285-294.

Jones AL, Bucholz RW, Bosse MJ, Mirza SK, Lyon TR, Webb LX, Pollak AN, Golden JD, Valentin-Opran A (2006) Recombinant BMP-2 and allograft compared with autogenous bone graft for reconstruction of diaphyseal tibial fractures with cortical defects. A randomized, controlled trial. J Bone Joint Surg Am 88: 1431-1441.

Joyce M (2005) Safety and FDA regulations for musculoskeletal allografts: perspective of an orthopaedic surgeon. Clin Orthop Relat Res 435: 22-30.

Kanczler JM, Oreffo RO (2008) Osteogenesis and angiogenesis: the potential for engineering bone. Eur Cell Mater 15: 100-114.

Karsenty G, Wagner EF (2002) Reaching a genetic and molecular understanding of skeletal development. Dev Cell 2: 389-406.

Khan Y, Yaszemski MJ, Mikos AG, Laurencin CT (2008) Tissue engineering of bone: material and matrix considerations. J Bone Joint Surg Am 90: 36-42.

Kolambkar YM, Dupont KM, Boerckel JD, Huebsch N, Mooney DJ, Hutmacher DW, Guldberg RE (2011) An alginate based hybrid system for growth factor delivery in the functional repair of large bone defects. Biomaterials 32: $65-74$.

Komori T (2010) Regulation of bone development and extracellular matrix protein genes by RUNX2. Cell Tissue Res 339: 189-195,
Le Nihouannen D, Komarova SV, Gbureck U, Barralet JE (2008) Bioactivity of bone resorptive factor loaded on osteoconductive matrices: stability post-dehydration. Eur J Pharm Biopharm 70: 813-818.

Lin CY, Chang YH, Lin KJ, Yen TC, Tai CL, Chen CY, Lo WH, Hsaio IT, Hu YC (2010) The healing of critical sized femoral segmental bone defects in rabbits using baculovirus-engineered mesenchymal stem cells. Biomaterials 31: 3222-3230.

McKee MD, Sodek J (2000) Bone matrix proteins. In: The Osteoporosis Primer (Henderson JE, Goltzman D, eds), Cambridge University Press, Cambridge, pp 46-63.

Miyagi Y, Chiu LL, Cimini M, Weisel RD, Radisic M, Li RK (2011) Biodegradable collagen patch with covalently immobilized VEGF for myocardial repair. Biomaterials 32: 1280-1290

Monfoulet L, Malaval L, Aubin JE, Rittling SR, Gadeau AP, Fricain JC, Chassande O (2010) Bone sialoprotein, but not osteopontin, deficiency impairs the mineralization of regenerating bone during cortical defect healing. Bone 46: 447-452.

Moore DC, Chapman MW, Manske D (1987) The evaluation of biphasic calcium phosphate ceramic for use in grafting long bone diaphyseal defects. J Orthop Res 5: 356-365.

Nauth A, Miclau T, Bhandari M, Schemitsch EH (2011) Use of osteobiologics in the management of osteoporotic fractures. J Orthop Trauma 25: S51-55.

Oetgen ME, Richards BS (2010) Complications associated with the use of bone morphogenetic protein in pediatric patients. J Pediatr Orthop 30: 192-198.

Roohani-Esfahani SI, Lu ZF, Ellis-Behnke R, Kaplan DL, Zreiqat H (2012) Effect of self-assembled nanofibrous silk/polycaprolactone layer on the osteoconductivity and mechanical properties of biphasic calcium phosphate scaffolds. Acta Biomater 8: 302-312.

Rootare HM, Powers JM, Craig RG (1978) Sintered hydroxyapatite ceramic for wear studies. J Dent Res 57: 777-783.

Serpooshan V, Julien M, Nguyen O, Wang H, Li A, Muja N, Henderson JE, Nazhat SN (2010) Reduced hydraulic permeability of three-dimensional collagen scaffolds attenuates gel contraction and promoted the growth and differentiation of mesenchymal stem cells. Acta Biomater 6: 3978-3987.

Shen YH, Shoichet MS, Radisic M (2008) Vascular endothelial growth factor immobilized in collagen scaffold promotes penetration and proliferation of endothelial cells. Acta Biomater 4: 477-489.

Soundrapandian C, Datta S, Kundu B, Basu D, Sa B (2010) Porous bioactive glass scaffolds for local drug delivery in osteomyelitis: development and in vitro characterization. AAPS PharmSciTech 11: 1675-1683.

Starman JS, Bosse MJ, Cates CA, Norton HJ (2012) Recombinant human bone morphogenetic protein-2 use in the off-label treatment of nonunions and acute fractures: a retrospective review. J Trauma Acute Care Surg 72: 676681.

Street J, Bao M, deGuzman L, Bunting S, Peale FV, Ferrara N, Steinmetz H, Hoeffel J, Cleland JL, Daugherty 
A, van Bruggen N, Redmond HP, Carano RA, Filvaroff EH (2002) Vascular endothelial growth factor stimulates bone repair by promoting angiogenesis and bone turnover. Proc Natl Acad Sci USA 99: 9656-9661.

Tay BK, Le AX, Gould SE, Helms JA (1998) Histochemical and molecular analyses of distraction osteogenesis in a mouse model. J Orthop Res 16: 636-642.

Uchida S, Sakai A, Kudo H, Otomo H, Watanuki M, Tanake M, Nagashima M, Nakamura T (2003) Vascular endothelial growth factor is expressed along with its receptors during the healing process of bone and bone marrow after drill-hole injury in rats. Bone 32: 491-501.

Valverde-Franco G, Liu H, Davidson D, Chai S, Valderrama-Carvajal H, Goltzman D, Ornitz DM, Henderson JE (2004) Defective bone mineralization and osteopenia in young adult FGFR3-/- mice. Hum Mol Genet 13: 271-284.

Zisch AH, Schenk U, Schense JC, Sakiyama-Elbert SE, Hubbell JA (2001) Covalently conjugated VEGFfibrin matrices for endothelialization. J Control Release 72: $101-113$

\section{Discussion with Reviewers}

M. Laschke: Rat tail collagen was used for the preparation of the scaffolds. Doesn't this induce the activation of the immune system after implantation into mice?

Authors: The short answer is no, we have never seen an immune reaction when rat tail collagen is used in mice. The sequence of Type I Collagen is highly conserved amongst species, which enables cross species transplantation in the absence of an immune reaction. In fact bovine collagen is used in many products that have been approved for human use.

M. Laschke: Do the authors feel that repetitive VEGF injections could further improve the efficacy of their approach? What application scheme would they suggest? Authors: Improved patient outcomes are associated with a reduction in the number and/or complexity of invasive procedures. To avoid repetitive injection of agents such as VEGF they could be cross-linked directly to the type I collagen or contained in particulate form for prolonged release from the scaffold. In this respect it will be crucial to establish the release kinetics and perform detailed dose/ response experiments for the therapeutic agent.

S. Verrier: Couldn't be the increase of thickness and volume due to a re-hydration of the collagen exposed to the body fluids?

Authors: As described in Serpooshan et al. (2010) (text reference) the dense collagen scaffold was generated through plastic compression and was therefore incapable of re-hydration. As the increase in thickness only occurred in the cell-seeded scaffolds after prolonged culture in osteogenic medium and in the presence of mineral, quantified by micro-CT, we attributed the increase to the mineral content. 